\title{
Editorial: Amoebae as Host Models to Study the Interaction With Pathogens
}

\author{
Sascha Thewes ${ }^{1 *}$, Thierry Soldati ${ }^{2 *}$ and Ludwig Eichinger ${ }^{3 *}$ \\ ${ }^{1}$ Department of Biology, Chemistry, Pharmacy, Institute for Biology - Microbiology, Freie Universität Berlin, Berlin, Germany, \\ ${ }^{2}$ Department of Biochemistry, Faculty of Science, University of Geneva, Sciences II, Geneva, Switzerland, ${ }^{3}$ Medical Faculty, \\ Center for Biochemistry, University Hospital Cologne, Cologne, Germany
}

Keywords: amoebae, host-pathogen-interaction, evolution, phagocytes, immune system

\section{Editorial on the Research Topic}

\section{Amoebae as Host Models to Study the Interaction With Pathogens}

Amoebae are eukaryotic microorganisms of great diversity. They do not form a single taxonomic group and are found among the protozoa, fungi, and algae. However, all amoebae are characterized by the amoeboid life style-the ability to change cell shape by extending and retracting pseudopods-and most amoebae can be considered as professional phagocytes, which feed on

\section{OPEN ACCESS}

Edited and reviewed by: Thomas Rudel,

Universität Würzburg, Germany

*Correspondence:

Sascha Thewes sascha.thewes@fu-berlin.de Thierry Soldati thierry.soldati@unige.ch Ludwig Eichinger

ludwig.eichinger@uni-koeln.de

Specialty section:

This article was submitted to

Bacteria and Host,

a section of the journal

Frontiers in Cellular and Infection

Microbiology

Received: 13 January 2019

Accepted: 13 February 2019

Published: 19 March 2019

Citation:

Thewes S, Soldati T and Eichinger $L$ (2019) Editorial: Amoebae as Host

Models to Study the Interaction With

Pathogens

Front. Cell. Infect. Microbiol. 9:47.

doi: 10.3389/fcimb.2019.00047 bacteria and other microorganisms by phagocytosis. But despite their impressive phagocytic activity, amoebae cannot degrade all microorganisms. Some resist the digestion and some even use amoebae as host cells for their own replication. Such microbial resistance is also observed in the complex relationship between phagocytes of the mammalian immune system and pathogenic bacteria. For example, Legionella pneumophila usually infects fresh-water amoebae, but can also use human alveolar macrophages to further its own replication. This similarity has led to the concept of "amoebae as training grounds for (intracellular) pathogenic bacteria," namely that pathogenic microorganisms can establish, select and "train" their virulence traits in free-living amoebae before being faced with phagocytic immune cells of animals (Molmeret et al., 2005). Recent studies using amoebae as host models for pathogens confirmed the broad plausibility of this concept. The amoebae used as host models predominantly belong to the phylum Amoebozoa, the closest phylum to fungi and animals, with their most prominent representatives from the genera Acanthamoeba and Dictyostelium. Both have been demonstrated to be useful host cells for the study of the complex interactions with bacterial pathogens such as Legionella, Mycobacterium, Salmonella, Francisella, and others (for reviews see Clarke, 2010; Bozzaro and Eichinger, 2011). Additionally, amoebae can also be used as model hosts for pathogenic fungi like Cryptococcus, Aspergillus, and Candida (Steenbergen et al., 2003; Chrisman et al., 2011; Hillmann et al., 2015; Mattern et al., 2015; Koller et al., 2016; Maisonneuve et al., 2016). These examples highlight the potential of amoebae as model hosts to study the interaction with a wide range of pathogenic microorganisms. In this Frontiers Research Topic a large range of aspects concerning amoebae as host cells for pathogens is covered.

\section{WHICH AMOEBAE CAN BE USED AS HOSTS?}

The currently most used and best-investigated amoebae hosts are Acanthamoeba castellanii and Dictyostelium discoideum. This is highlighted in a review by Swart et al. The authors summarize comprehensively the current knowledge on the interaction of $L$. pneumophila with its natural host A. castellanii and the versatile model host $D$. discoideum. The potential of $D$. discoideum as a host 
model to study different cellular aspects of the interaction with (pathogenic) bacteria is also presented in a method-oriented article by Meena and Kimmel. The authors share a range of protocols using D. discoideum to study chemotaxis, phagocytosis, and macropinocytosis.

Further amoebae are used to study specific aspects of the interaction with various pathogens. Diesend et al. summarize the current knowledge about the infection of free-living amoebae with giant viruses. Here, the best-studied amoeba is Acanthamoeba polyphaga, which can be infected by a mimivirus. For the study of the interaction of amoebae with pathogenic fungi, the amoebal repertoire is currently the largest. In a review by Novohradská et al. the authors describe the amoebal species that have been used to study fungal interactions. Beside $A$. castellanii and $D$. discoideum, amoebae such as the common water contaminant Vermamoeba vermiformis, Protostelium mycophagum, and other mycophagous soil amoebae from the genera Thecamoeba, Arachnula, and Vampyrella have been used to study the interaction with human-, plant-, and entomopathogenic fungi.

\section{WHICH PATHOGENS CAN BE STUDIED USING AMOEBAE?}

The range of microorganisms, which can be studied using different amoebae, is not limited to a specific group. Amoebae are already well-established model systems to study the interaction with bacteria. The most prominent bacteria studied using $A$. castellanii and $D$. discoideum are currently from the genera Legionella and Mycobacterium (see above and reviews by Swart et al.; Cardenal-Muñoz et al.). Two original research articles also highlight this fact. Buracco et al. show that iron depletion or overload of $D$. discoideum cells impacts on the intracellular growth of Legionella, whereas changes in zinc and copper concentrations do not have any effect. Concerning mycobacteria, Samba-Louaka et al. show that free-living amoebae might serve as a reservoir for Mycobacterium avium subsp. paratuberculosis. The authors found that on farms, where cattle were infected with $M$. avium subsp. paratuberculosis, the bacteria were also found in environmental samples containing amoebae of the poorly described Rosculus genus. Salmonella enterica serovar Typhimurium is a further example of bacteria that can be studied using amoebae as hosts. Varas et al. show that inorganic polyphosphate (polyP) is crucial for the virulence of these bacteria. Mutation of the polyP kinase $(p p K)$ gene in $S$. Typhimurium affects the ability of intracellular replication. Finally, the spectrum of pathogenic bacteria is constantly expanding. Recently, it has been shown that Bordetella bronchiseptica can use amoebae as an environmental niche and transmission vector (Taylor-Mulneix et al., 2017). In a perspective article Taylor-Mulneix et al. now ask whether amoebae are the missing link for the evolution of Bordetellae from environmental microbes to human respiratory pathogens. In this article the concept of amoebae as "training ground" for human pathogens is picked up again. Brock et al. underline this ecological relevance for amoebae as a reservoir for (pathogenic) bacteria in an original research article. The authors isolated environmental samples of $D$. discoideum and found that one third of the wild isolates carried one to six bacterial species per fruiting body. The majority of the isolated bacteria were from the genus Proteobacteria but also Actinobacteria, Bacteriodetes, and Firmicutes have been identified. Many of the bacterial genera isolated in this study include species that are implicated in causing diseases, such as Brucella, Nocardia, pathogenic E. coli, Shigella, and Staphylococcus, suggesting that freeliving amoebae can serve as reservoirs for animal and human pathogenic bacteria.

In recent years the use of amoebae as host cells has been expanded to study interactions with different fungi. Novohradská et al. sum up the current knowledge regarding filamentous fungi (human-, plant-, and entomopathogenic). Watkins et al. show in their original research article that the environmental pathogenic yeast Cryptococcus neoformans escapes from $D$. discoideum cells after phagocytosis by two different pathways: canonical exocytosis and non-lytic release by vomocytosis, which are the same mechanisms for $C$. neoformans to escape from macrophages.

Last but not least, amoebae as host cells are not restricted to bacteria or fungi. They can also be used to study the interaction with viruses such as the giant mimivirus (Diesend et al.).

All these examples show that different amoebae can be used with a plethora of microorganisms to learn more about the relationship and interaction from an ecological, evolutionary, and medical point of view.

\section{WHICH ASPECTS OF PHAGOCYTOSIS AND KILLING BY THE AMOEBAE ARE IN COMMON WITH MAMMALIAN PHAGOCYTES?}

Although amoebae are used as host model systems to study interactions with pathogenic microorganisms, one major drawback is that most amoebae grow at environmental temperatures and are usually not able to grow at elevated temperatures such as the human body temperature. However, mammalian phagocytes and amoebae share many conserved cellular and molecular processes concerning phagocytosis and intracellular killing of pathogens. In a mini-review Mori et al. compare and discuss the roles for mammalian and D. discoideum coronins in the trafficking and survival of intracellular pathogens. Although many aspects of the regulation and function of coronins are still elusive, a conserved role for coronins in $D$. discoideum and mammals can be delineated. Similarly, mechanisms of host defense processes and intracellular virulence of Legionella and Mycobacterium are surprisingly highly evolutionarily conserved between animal macrophages and amoebae, as exhaustively reviewed by Swart et al. and Cardenal-Muñoz et al. Additionally, Watkins et al. show that vomocytosis of Cryptococcus cells is mechanistically conserved between amoebae and mammalian macrophages. Further, Novohradská et al. nicely compare the parallel events in the phagocytic processing of Aspergillus conidiae in mammalian 
macrophages and amoebae, again supporting the concept of amoebae as training ground for pathogens.

Amoebae are also interesting models to study the early evolution of cell-autonomous, innate immunity (Leippe, 1999), which is reflected in an original research article by Dhakshinamoorthy et al. who investigated the pore-forming saposin-like proteins in the defense against bacterial infection. Such studies show that the limit of amoebae as host models systems is not reached yet.

\section{CONCLUSIONS}

It is undisputable that phagocytosis and intracellular processing of pathogenic microorganisms is not identical in every aspect in animal phagocytes and amoebae. However, this research topic and abundant recent publications have illustrated that numerous aspects of these processes are evolutionarily conserved between amoebae and animal phagocytes. Further, the advantages of amoebae as host cells are obvious. Amoebae can be cultivated easily in the lab and-especially in the case of D. discoideumcan be easily genetically manipulated. Additionally, many other tools for molecular and cell biology have been established for the use with amoebae.

\section{REFERENCES}

Bozzaro, S., and Eichinger, L. (2011). The professional phagocyte Dictyostelium discoideum as a model host for bacterial pathogens. Curr. Drug Targets 12, 942-954. doi: 10.2174/138945011795677782

Chrisman, C. J., Albuquerque, P., Guimaraes, A. J., Nieves, E., and Casadevall, A. (2011). Phospholipids trigger Cryptococcus neoformans capsular enlargement during interactions with amoebae and macrophages. PLoS Pathog. 7:e1002047. doi: 10.1371/journal.ppat.1002047

Clarke, M. (2010). Recent insights into host-pathogen interactions from Dictyostelium. Cell. Microbiol. 12, 283-291. doi: 10.1111/j.1462-5822.2009.01413.x

Diop, E. A., Queiroz, E. F., Kicka, S., Rudaz, S., Diop, T., and Wolfender, J. L. (2018). Survey on medicinal plants traditionally used in Senegal for the treatment of tuberculosis (TB) and assessment of their antimycobacterial activity. J. Ethnopharmacol. 216, 71-78. doi: 10.1016/j.jep.2017.12.037

Harrison, C. F., Chiriano, G., Finsel, I., Manske, C., Hoffmann, C., Steiner, B., et al. (2015). Amoeba-based screening reveals a novel family of compounds restricting intracellular Legionella pneumophila. ACS Infect. Dis. 1, 327-338. doi: 10.1021/acsinfecdis.5b00002

Hillmann, F., Novohradska, S., Mattern, D. J., Forberger, T., Heinekamp, T., and Brakhage, A. A. (2015). Virulence determinants of the human pathogenic fungus Aspergillus fumigatus protect against soil amoeba predation. Environ. Microbiol. 17, 2858-2869. doi: 10.1111/1462-2920.12808

Kicka, S., Trofimov, V., Harrison, C., Ouertatani-Sakouhi, H., McKinney, J., Scapozza, L., et al. (2014). Establishment and validation of whole-cell based fluorescence assays to identify anti-mycobacterial compounds using the Acanthamoeba castellanii-Mycobacterium marinum host-pathogen system. PLoS ONE 9:e87834. doi: 10.1371/journal.pone.0087834

Koller, B., Schramm, C., Siebert, S., Triebel, J., Deland, E., Pfefferkorn, A. M., et al. (2016). Dictyostelium discoideum as a Novel Host System to Study the interaction between phagocytes and yeasts. Front. Microbiol. 7:1665. doi: $10.3389 /$ fmicb.2016.01665

Leippe, M. (1999). Antimicrobial and cytolytic polypeptides of amoeboid protozoa-effector molecules of primitive phagocytes. Dev. Comp. Immunol. 23, 267-279. doi: 10.1016/S0145-305X(99)00010-5

Maisonneuve, E., Cateau, E., Kaaki, S., and Rodier, M. H. (2016). Vermamoeba vermiformis-Aspergillus fumigatus relationships and
Such powerful systems will allow to further our understanding of the biology and evolution of host-pathogen interactions. This will constitute the basis for the development of novel anti-infection therapies as it has been shown already for Mycobacterium and Legionella (Kicka et al., 2014; Harrison et al., 2015; Ouertatani-Sakouhi et al., 2017; Diop et al., 2018; Trofimov et al., 2018). In summary, the future for amoebae as model systems to unravel host-pathogen interactions has just begun.

\section{AUTHOR CONTRIBUTIONS}

All authors listed have made a substantial, direct and intellectual contribution to the work, and approved it for publication.

\section{FUNDING}

ST received funding from the Freie Universität Berlin. The TS lab is supported by grants from the Swiss National Science Foundation (310030_149390 and 310030_169386) and the SystemsX.ch initiative grant HostPathX. LE acknowledges support by the Deutsche Forschungsgemeinschaft (CRC670, TP01) and by Köln Fortune.

comparison with other phagocytic cells. Parasitol. Res. 115, 4097-4105. doi: 10.1007/s00436-016-5182-3

Mattern, D. J., Schoeler, H., Weber, J., Novohradska, S., Kraibooj, K., Dahse, H. M., et al. (2015). Identification of the antiphagocytic trypacidin gene cluster in the human-pathogenic fungus Aspergillus fumigatus. Appl. Microbiol. Biotechnol. 99, 10151-10161. doi: 10.1007/s00253-015-6898-1

Molmeret, M., Horn, M., Wagner, M., Santic, M., and Abu Kwaik, Y. (2005). Amoebae as training grounds for intracellular bacterial pathogens. Appl. Environ. Microbiol. 71, 20-28. doi: 10.1128/AEM.71.1. 20-28.2005

Ouertatani-Sakouhi, H., Kicka, S., Chiriano, G., Harrison, C. F., Hilbi, H., and Cosson, P. (2017). Inhibitors of Mycobacterium marinum virulence identified in a Dictyostelium discoideum host model. PLoS ONE 12:e0181121. doi: 10.1371/journal.pone.0181121

Steenbergen, J. N., Nosanchuk, J. D., Malliaris, S. D., and Casadevall, A. (2003). Cryptococcus neoformans virulence is enhanced after growth in the genetically malleable host Dictyostelium discoideum. Infect. Immun. 71, 4862-4872. doi: 10.1128/IAI.71.9.4862-4872.2003

Taylor-Mulneix, D. L., Bendor, L., Linz, B., RiveraI, Ryman, V. E., Dewan, K. K. et al. (2017). Bordetella bronchiseptica exploits the complex life cycle of Dictyostelium discoideum as an amplifying transmission vector. PLoS Biol. 15:e2000420. doi: 10.1371/journal.pbio.2000420

Trofimov, V., Kicka, S., Mucaria, S., Hanna, N., Ramon-Olayo, F., Del Peral, L. V., et al. (2018). Antimycobacterial drug discovery using Mycobacteria-infected amoebae identifies anti-infectives and new molecular targets. Sci. Rep. 8:3939. doi: 10.1038/s41598-018-22228-6

Conflict of Interest Statement: The authors declare that the research was conducted in the absence of any commercial or financial relationships that could be construed as a potential conflict of interest.

Copyright (c) 2019 Thewes, Soldati and Eichinger. This is an open-access article distributed under the terms of the Creative Commons Attribution License (CC BY). The use, distribution or reproduction in other forums is permitted, provided the original author(s) and the copyright owner(s) are credited and that the original publication in this journal is cited, in accordance with accepted academic practice. No use, distribution or reproduction is permitted which does not comply with these terms. 